\title{
THE GENERAL PRODUCT OF TWO FINITELY GENERATED ABELIAN GROUPS
}

\author{
EUGENE SCHENKMAN ${ }^{1}$
}

Ito [2] has proved that if a group $G$ is the general product $A B$ where $A$ and $B$ are finite abelian groups then $G$ has a normal subgroup contained either in $A$ or in $B$ and a normal subgroup containing $A$ or $B$. We use an idea of P. M. Cohn [1] to extend Ito's Theorem to the case where $A$ and $B$ are finitely generated. As a consequence we see that the general product of two finitely generated abelian groups is polycyclic and we incidentally also obtain an easier proof of Cohn's results. Our main theorem is as follows.

Theorem. Let $G=A B$ with $A$ and $B$ finitely generated abelian subgroups. Then

(I) $G$ contains a nontrivial normal subgroup contained either in $A$ or in $B$ and

(II) $G$ contains a proper normal subgroup containing $A$ or $B$.

Proof of (I). Let $A^{*}$ denote $A \cap G^{\prime}\left(G^{\prime}\right.$ is the commutator subgroup $[G, G])$ and let $B^{*}$ denote $B \cap G^{\prime}$. If $A^{*}>1$ or $B^{*}>1$, then (I) is true, as Ito has already pointed out. Accordingly we assume that $A^{*}=B^{*}=1$ and also that $G$ is infinite in view of Ito's Theorem. Let $A_{0}$ be the set of elements $a \in A$ such that there is an element $b \in B$ with $a b \in G^{\prime}$, and let $B_{0}$ be the set of elements $b \in B$ with an $a \in A$ so that $a b \in G^{\prime}$. We shall show that $A_{0}$ and $B_{0}$ are isomorphic subgroups. Indeed, since $A^{*}=B^{*}=1$ it is clear that if $a b$ and $a b^{\prime} \in G^{\prime}$ with $a \in A_{0}$ and $b, b^{\prime} \in B_{0}$, then $b^{-1} a^{-1} a b^{\prime}=b^{-1} b^{\prime} \in B^{*}=1$ so that $b=b^{\prime}$. Thus there is a well-defined map $\phi$ from $A_{0}$ onto $B_{0}$ so that if $a \in A_{0}$, then $a \phi \in B_{0}$ and $a(a \phi) \in G^{\prime}$, and for later reference it will be convenient to define the map $\psi$ from $A_{0}$ to $G^{\prime}$ as follows: $a \psi=a a \phi$; it is easy to check that $\phi$ is one-one and $\psi$ is single valued. Then if $a b$, $a^{\prime} b^{\prime} \in G^{\prime}$ with $a, a^{\prime} \in A_{0}$ and $b, b^{\prime} \in B_{0}$, it follows that $a b\left(b^{-1} a^{\prime} b^{\prime} b\right)$ $=a a^{\prime} b b^{\prime} \in G^{\prime}$; thus $A_{0}$ and $B_{0}$ are semigroups and $\phi$ is a semigroup isomorphism. Finally $\left(b a b b^{-1}\right)^{-1}=a^{-1} b^{-1}$, and hence $A_{0}$ and $B_{0}$ are isomorphic groups as we wished to show and $G^{\prime}$ is the set of elements $a(a \phi)$ with $a \in A_{0}, a \phi \in B_{0}$.

Received by the editors February 29, 1968.

${ }_{1}^{1}$ The author is indebted to D. Hertzig for some useful comments during the preparation of this paper, to $\mathrm{O}$. Kegel for pointing out Corollary 1, and to the National Science Foundation for support. 
Now we shall show that if for $i=1,2, M_{i}$ are normal subgroups of $G$ with $M_{2} \leqq M_{1} \leqq G^{\prime}$, then $M_{i} \psi^{-1}=A_{i}$ are subgroups and the index $\left|M_{1}: M_{2}\right|$ is equal to the index $\left|A_{1}: A_{2}\right|$. Indeed the fact that $A_{i}$ is a subgroup follows from the argument above with $M_{i}$ in place of $G^{\prime}$. Then for $a, a^{\prime} \in A_{1}$ the cosets $a A_{2}, a^{\prime} A_{2}$ are equal if and only if the cosets $a(a \phi) M_{2}=a^{\prime}\left(a^{\prime} \phi\right) M_{2}$. For $a A_{2}=a^{\prime} A_{2}$ if and only if $a^{-1} a^{\prime} \in A_{2}$ which holds if and only if $(a \phi)^{-1} a^{-1} a^{\prime}\left(a^{\prime} \phi\right)$ or $a^{-1} a^{\prime}\left(a^{\prime} \phi\right)(a \phi)^{-1} \in M_{2}$ (since $\left.M_{2} \triangleleft G\right)$; and this holds if and only if $a^{\prime}\left(a^{\prime} \phi\right) M_{2}=a(a \phi) M_{2}$. Hence $\left|M_{1}: M_{2}\right|=\left|A_{1}: A_{2}\right|$ as was to be shown.

It follows in particular that the order of a finite subgroup of $G^{\prime}$ normal in $G$ is bounded by the order $t$ of the torsion subgroup of $A_{0}$ (or of $B_{0}$ ). Furthermore, since factoring out a normal subgroup of $G$ contained in $G^{\prime}$ does not alter the hypotheses $A^{*}=1=B^{*}$, it follows that if $M / M_{2}$ is finite with $M_{2} \leqq M_{1} \leqq G^{\prime}$ as before, then the order of $M_{1} / M_{2}$ is bounded by $t$. Consequently there is a minimal normal subgroup $M$ of $G$ contained in $G^{\prime}$; for in any decreasing sequence of normal subgroups of $G, G^{\prime}=M_{0}>M_{1}>\cdots>M_{i}>\cdots>M_{j}>\cdots$, there can be at most as many infinite factors $M_{i} / M_{i+1}$ as the torsionfree rank of $A_{0}$ and the finite indices $\left|M_{i}: M_{j}\right|$ are bounded by $t$.

Let $A_{M}$ denote $M \psi^{-1}$ and $B_{M}$ denote $A_{M} \phi$. Modulo $M, A_{M}=B_{M}$ and hence $A_{M}$ is central in $G$ (since $G=A B$ ). Thus $A_{M} B_{M} \triangleleft G$ and $M=A_{M} B_{M} \cap G^{\prime}$. If $A_{M} B_{M}$ is not abelian there is an $a^{-1} b \in M$ with $a \in A_{M}$ so that $\left[a, B_{M}\right] \neq 1$. Let $S$ be the subgroup generated by $a$ and $G^{\prime}$. Then $S$ is normal in $G$ and so also is the commutator subgroup $S^{\prime}$. Furthermore $S^{\prime} \leqq G^{\prime} \cap A_{M} B_{M}=M$ since $a \in A_{M}$ and $A_{M} B_{M} \triangleleft G$. Hence by the minimality of $M, S^{\prime}=M$. Now in general if $S$ is the extension of a normal abelian subgroup $C$ by a cyclic group generated by $a$, then $S^{\prime}$ is the set $T$ of commutators $\{[a, c], c \in C\}$. For $\left[a, c_{1} c_{2}\right]=\left[a, c_{1}\right]\left[a, c_{2}\right]$ so that $\left[a, c^{-1}\right]=[a, c]^{-1}=[c, a]$ and hence $T$ is a subgroup. Furthermore, from the fact that $L=\left[a^{-1} a, c\right]$ $=\left[a^{-1}, c\right]^{a}[a, c]=\left[a^{-1}, c\right]\left[\left[a^{-1}, c\right], a\right][a, c]$, we see that $\left[a^{-1}, c\right]$ is of the form $\left[a, c^{\prime}\right]$ for some $c^{\prime} \in C$ and hence $T \triangleleft S$. Since $S$ modulo $T$ is clearly abelian it follows that $S^{\prime}=T$ as we wished to show. Accordingly, in the case under consideration,

$$
S^{\prime}=M=\left[a, G^{\prime}\right]=\left\{\left[a, b_{0}\right], b_{0} \in B_{0}\right\} .
$$

Hence since $a^{-1} b \in M$, there is $b_{0} \in B_{0}$ so that $a^{-1} b=\left[a, b_{0}\right]$ and hence $a^{b_{0}}=b$. Thus $a$ and $b$ are conjugates, and if $C_{a}, C_{b}$ denote the centralizers respectively of $a$ and $b$, then $A \leqq C_{a}, B \leqq C_{b}$ and $G=A B=C_{a} C_{b}$. Since $C_{a}$ and $C_{b}$ are conjugates, $G$ can equal $C_{a} C_{b}$ only if $C_{a}=G$ (in general if $G=F H$ with $F=H^{o}, g=h f^{-1}, h \in H, f \in F$, then $F=F^{f}=H^{h}$ $=H$ and $G=F)$. Consequently, $a$ is central in $G$ contrary to the fact that $\left[a, B_{M}\right] \neq 1$. 
We conclude that $A_{m} B_{m}$ is abelian and hence that $M$ is isomorphic to $A_{M}$. Then from its minimality $M$ is finite. By factoring out $M$ we see that modulo $M$, a minimal normal subgroup of $G$ contained in $G^{\prime}$, is again finite. Since the chain is finite, we may conclude that $G^{\prime}$ is finite. Since $G^{\prime}$ is finite, each $g \in G$ has a finite number of conjugates and hence the centralizer $C_{g}$ has finite index in $G$. Then since $G$ is finitely generated, the center of $G$ has finite index in $G$ and hence intersects $A$ or $B$ nontrivially ( $G$ is infinite by assumption). Thus $A$ or $B$ has a central subgroup of $G$ and the proof of (I) is complete.

REMARK. A proof of (I) for $G$ finite can be based on the fact that $G$ has exactly one conjugacy class of Carter (i.e. self-normalizing nilpotent) subgroups. For then not both $A$ and $B$ can be Carter subgroups and hence, without loss of generality, $A$ is properly contained in its normalizer $N$. Let $D$ denote $N \cap G^{\prime}$. If $D=1$, then $N \cong N G^{\prime} / G^{\prime}$ and is abelian; while if $D>1$, then $[A, D] \leqq A \cap G^{\prime}=1$ and hence $A D$ is abelian. In either case $N$ contains an abelian subgroup properly containing $A$ and hence an element $a b$ with $a \in A$, $b \in B, b \neq 1$ such that $[b, A]=1$ so that $(b)$ is central in $G$.

Proof of (II). We proceed by induction first on the sum of the torsion-free ranks of $A$ and $B$ and then on the sum of the orders of the torsion subgroups of $A$ and $B$. In view of (I) there is a normal subgroup $N$ of $G$ contained without loss of generality in $A$. Then $G / N$ $=A / N \cdot B N / N$ and by the induction assumption (either the torsionfree rank or the order of the torsion subgroup of $A / N$ is less than the corresponding quantity of $A$ ), there is a subgroup $M$ of $G$ containing $N$ so that $M / N$ is a proper normal subgroup of $G / N$ containing either $A / N$ or $B N / N$. It follows that $M$ is a proper normal subgroup of $G$ containing one of the subgroups $A$ or $B$ and (II) is proved.

An induction as in the proof of (II) above easily gives the following.

Corollary 1. If $G$ is the general product $A B$ with $A$ and $B$ finitely generated abelian subgroups, then $G$ is polycyclic; that is, $G$ is solvable and satisfies the maximal condition for subgroups.

Corollary 2. If $G$ is the general product $A B$ with $A$ and $B$ cyclic, then $G$ is supersolvable.

\section{BIBLIOGRAPHY}

1. P. M. Cohn, A remark on the general product of two infinite cyclic groups, Arch. Math. 7 (1956), 94-99.

2. N. Ito, Ueber das Produkt von zwei abelschen Gruppen, Math. Z. 62 (1955), 400401.

Purdue University 